\title{
Study on Quality Control of Drilling Fluid
}

\section{Introduction}

Drilling fluid plays an important part in the drilling engineering. However, because of the influence of present situation of domestic drilling teams of farmers and lack of professional and technical training of drilling skilled workers. Drilling fluid has not yet been paid sufficient attention and the configure and the usage of the fluid are in mess in the specific construction. People always only pursue economic interests, but they don't have an efficient quality control on each section of the drilling fluid, which leads to a lack of professional personnel in the drilling construction, a lack of instruments and a perfect flushing fluid management system. In a way, the drilling quality and efficiency decrease, but the cost of it increases. Sometimes it is hard to keep on drilling. After years of research and development, the fluid additive has developed from only using drilling bits to using the drilling fluid system to meet needs of every aspect. Such as, the polymer fluid with low viscosity, low friction and low solid phase for the fast drilling, the fluid for preventing obstruction, the fluid for preventing collapse of different kinds of rocks and the saturated salt-water drilling fluid for drilling salt rocks, which come to a relatively completed fluid system. But recent years, the fluid is lack of the scientific nature and extensive in the preparation, usage and management of the drilling construction. We need to change the backwards present situation of construction technology of drilling works, improve the quality control of drilling fluid and increase the construction quality to lay the foundation for solving complicate problems.

\section{Problems on the quality control of drilling fluid}

Because of the change of management system and the decrease of drilling management work, the quality management and control of drilling fluid go backwards seriously, which shows as follows:

\subsection{Lack of knowledge in guiding ideology.}

The drilling construction manager only see the increase of material and labor cost by providing highquality fluid and strict management but they don't pay attention to benefits of integrated technology, such as decreasing accidents in drill-hole, increasing the drilling efficiency, and decreasing the wear of drill pipes.

\subsection{Lack of professional personnel.}

Operators work by themselves and skillful workers teach and help new workers. For the whole industry, there is not a leading and demonstration team.

\subsection{Lack of equipment configuration and test instruments.}

At present, most of equipment doesn't go with test instruments, and the design of fluid is lack of scientific. The design of fluid is even farther from the actual performance indicators.

\subsection{The level of field fluid quality control is extremely low.}

There are no positions for machine washing fluid management and no tests for field fluid property. The fluid purifying equipment isn't used and sometimes the fluid circulation systems can't meet the requirements.

All the problems cause a great effect on the drilling production. There are too many accidents in drill-hole and too long time to stay in. But the efficiency is extremely low and the quality of drilling holes is bad. All of those must be solved.

\begin{abstract}
Drilling fluid is an important construction technique in the drilling engineering. However, because of the influence of present situation of domestic drilling geological, drilling fluid has not yet been paid sufficient attention. During the construction process, there is not enough professional personnel and apparatus, the fluid recipes are in mess, and they can't meet different kinds of formations, which cause that the efficiency of the drilling work is low and accidents in the hole happens frequently. This passage which is based on the characteristics and principles of quality control in each section of drilling fluid studies on the quality control, the system composition and how to ensure quality in details. It is also hoped to have the certain value and significance for the future quality control of drilling fluid.
\end{abstract}

Key words: Drilling fluid quality control; characteristics, principles; system

Published online: 31st January, 2018

\section{Characteristics and} principles of the fluid quality control

2.1 Characteristics of the fluid quality control

2.1.1 Many factors on the effect of the fluid quality control. The fluid is widely influenced by drilling methods in application fields, levels of operators, instruments for dealing with the fluid, operating processes, types and material of the fluid, drilling formations and environments. And all the factors make a certain effect on the fluid quality control, some has a direct effect and some has an indirect effect.

2.1.2 Dynamic and real-time of the fluid quality control. 
The fluid quality is a dynamic process which is changing as drilling, it needs checking and adjusting ye time to keep a stable design performance. The fluid is designed according to the drilling technology, the known formations, and the economic benefits. But the drilled formations are greatly uncertain, especially the fragmentation. Different formations need different kinds of fluid, which are on the basis of real-time formations to adjust, even change the fluid.

\subsection{Principles of the fluid quality control}

2.2.1 Take comprehensive economic benefits as the center. The purpose of the fluid quality control is to produce the largest economic benefits for the drilling production. But the proper purpose is to meet the drilling needs to the fluid property. And the key is to require high technology people.

2.2.2 People are the first priority of the quality control. People are the main objects to keep and control the fluid quality, who contain technicians, operators and managers. The occupational level and the professional competence of all kinds of operators play a more or less part in the fluid quality. So as checking the fluid quality, people must be emphasized. Control the whole literacy competency of all operators in order to improve the maintain and control of the fluid quality.

2.2.3 Design is the key. There are so many factors to think about in every section of the fluid quality and make comprehensive control measures and recommended solutions to the impossibly occurred problems which influence the mud quality.

2.2.4 Take < Drilling fluid technology management approach > as rules. < Drilling fluid technology management approach > is a professional technology management file mixed scientific technology and practical experience.

\section{The fluid quality control measures in every section}

3.1 Strengthen the quality control of management

Drilling field fluid management is the basis for the fluid quality control, and it is also the direct reflection of the fluid quality to maintain control. All of these depend on people. So people are the main objects to carry out the fluid quality control. All the controls without people's check can't maintain the production quality, though there are too many advanced equipments and excellent materials.

For the specific situations, people's check should lead people to form a sense of quality conscious, implement responsibilities and strengthen the supervision of the duties. The specific structure chart as Picture 1.

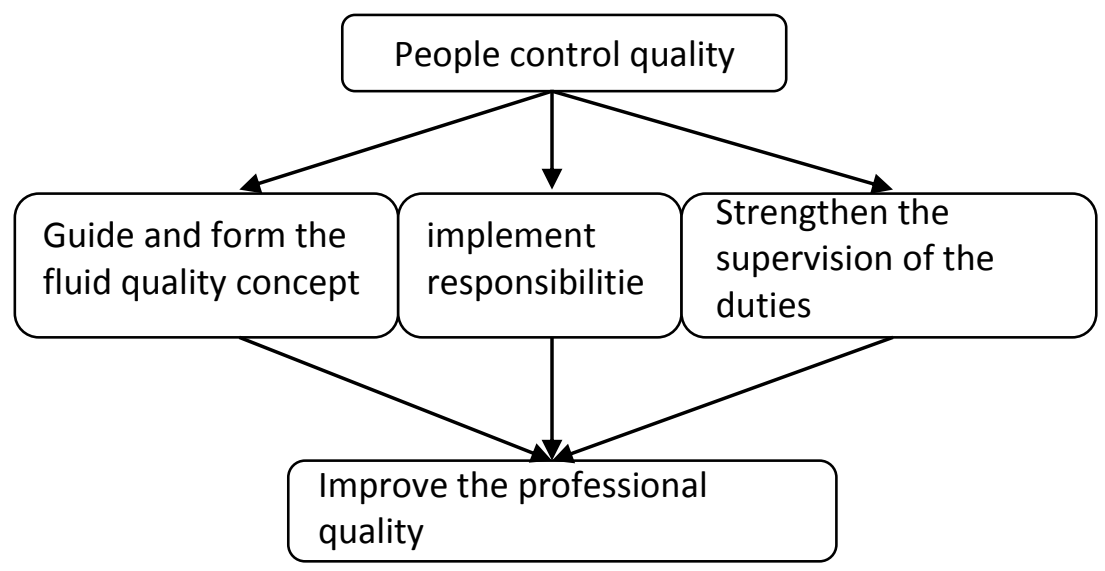

Picture 1. Structure chart of people's control of the quality

(1) Guide operators to form the fluid quality concept.

According to this point, lead operators to renew their opinions of the fluid property and form objective slurry quality, and the relative operators should know that the fluid is the key section of the drilling construction. Otherwise, put the quality first through the whole process and every section must follow it.

(2) Implement responsibilities.

Strengthen the quality concept of the drilling operators and implement responsibilities as well, which means every operator has his own duty.

(3) Strengthen the supervision of the duties.
Apart from implementing responsibilities, strengthen the supervision of the duties. However, the whole implementation is not perfect, which dues to the lack of effective supervision work. According to that, it is necessary to make a drilling slurry supervision system. Relative leaders check in the field regularly and put it into 
the whole supervision work.

In a word, people control the quality, which is not staged work, and put it into the whole supervision work. Organize operators to take part in quality control trainings and check the implementation regularly.

3.2 The fluid quality control in the preparation stage

Build a simple laboratory before the drilling construction, and complete all the testing instruments for the construction site. Research and survey the geologic configuration and structures to decide the type of the fluid. Design the fluid recipe, methods and processes through experiments and decide the fluid material.

3.2.1 The quality control of building and managing a slurry laboratory

The fluid property changes gradually in the loop running course, which requires to monitor the property parameter in real time, and adjust the fluid property in time according to the real complicated formations. However, before the adjustment, simulation experiments must be done to judge the efficiency of the design. So it is necessary to build a slurry laboratory. At the same time, the laboratory plays a very important part of studying new technology and cultivate drilling technical personnel. So it is also necessary to control the management.

All the testing instruments for the construction site show in and the common equipments shows in Picture 2.

\begin{tabular}{|c|l|c|l|}
\hline $\begin{array}{c}\text { Numbe } \\
r\end{array}$ & \multicolumn{1}{|c|}{ Equipment } & Quantity & Notes \\
\hline 1 & Densimeter (range<2.0g/cm ${ }^{3}$ ) (set) & 2 & \\
\hline 2 & $\begin{array}{l}\text { Fan's \& Marsh Funnel Viscosity } \\
\text { (set) }\end{array}$ & 2 & \\
\hline 3 & ZNN-D6 (set) & 1 & \\
\hline 4 & API Filter Press (suite) & 1 & \\
\hline 5 & Relative Content Analyzer (set) & 1 & \\
\hline 6 & Sediment Analyzer (suite) & 1 & \\
\hline 7 & PH Meter/ Test Paper (suite) & 1 & \\
\hline 9 & E-P Tester (suite) & 1 & \\
\hline 10 & Stopwatch (set) & 2 & \\
\hline 11 & 2000ml Drilling Counting Cup (set) & 1 & Enamel or stainless \\
& & 1 & \\
\hline 12 & High Mixer (suite) & 1 & \\
\hline 13 & Electron Balance (0.1g) (set) & & \\
\hline
\end{tabular}

Table 1. All the testing instruments for the construction site 

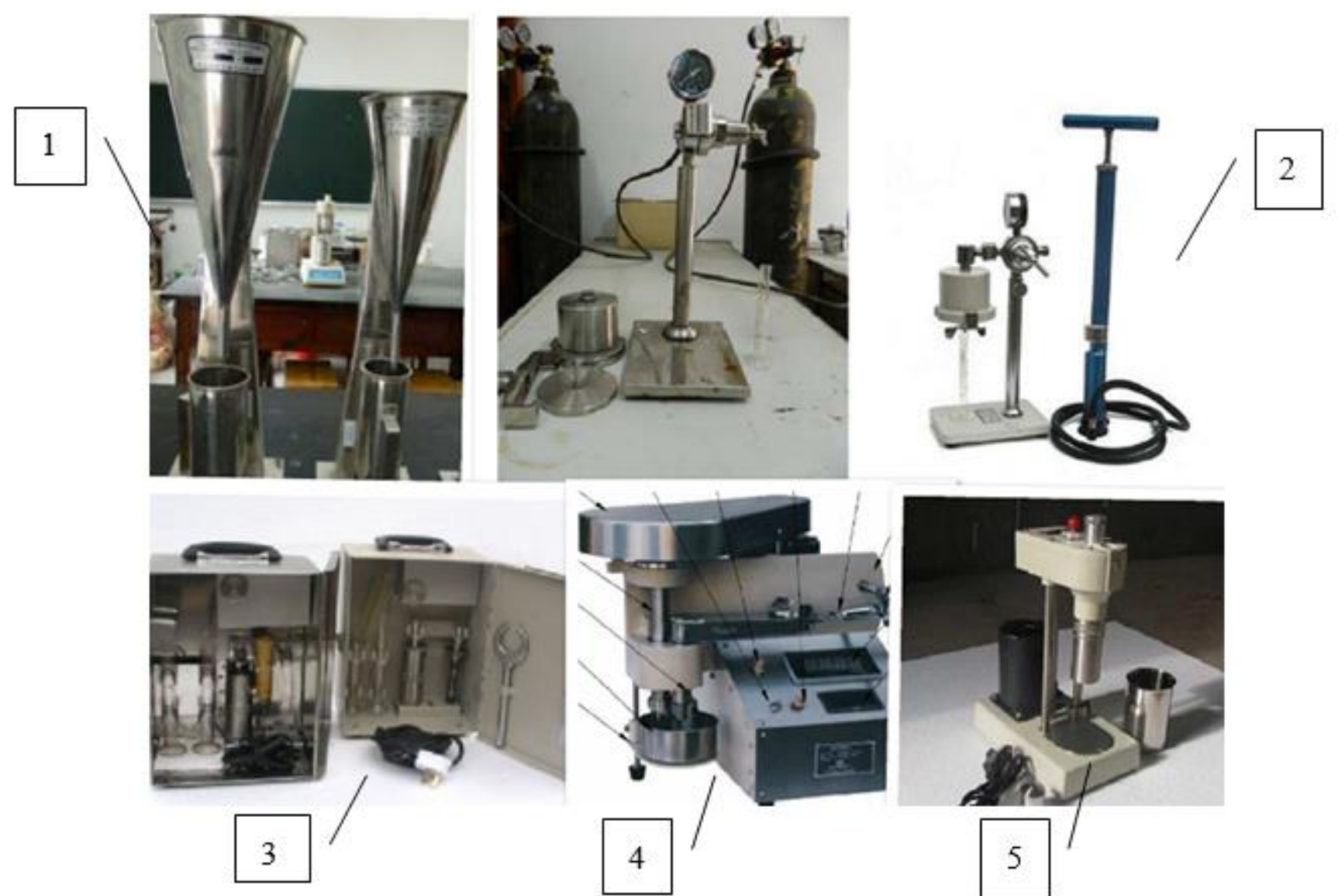

Picture 2. Common equipments: 1. Funnel Viscosity 2. Medium Pressure Filter Tester 3. Solid Content Analyzer 4. EP-B Tester 5. Rotational Viscosity

The daily maintenance of equipments is the key section of the laboratory management. The purpose is to ensure the function and detection accuracy of the equipments, and increase the use time of the equipments. So it is very necessary to make the daily maintenance system of the slurry laboratory equipments, which plays an important part in the daily slurry maintenance.

\subsubsection{Control the fluid quality}

\section{(1) Design basis.}

The drilling fluid is designed on the main basis of the drilling technique(such as the reverse circulation drilling, the wire-line core drilling, the foam drilling, the hydro-hammer drilling and the drilling structure and depth), the formation geological conditions(such as the geologic age, the reservoir stress, the rock lithology and cementation, the integrity of formations, the thickness, the depth, the extent of porosity and the temperature gradient), the seam protection, the environment protection and the circulatory conditions, which have the direct effect on choices of the types and the performance indicators of the fluid. All kinds of conditions should select the appropriate fluid design.

\section{(2) Design principles.}

First, according to the related technical specifications, regulations and standard, base the design of drilling fluid on the geological design, drilling engineering design and other related information. Second, go to the field to collect various formation samples of affecting drilling security as possible. Third, make related drilling fluid measures on the basis of anglicizing the security quality and efficiency. Fourth, design the recipe by the sample immersion experiments and choose the best scheme. Fifth, adjust the scheme immediately when you find the scheme can't meet the drilling need as the formation geological conditions are changing. Sixth, when there is contradiction in the fluid design, grasp the main problems, take into account secondary problems, and take integrated care of comprehensive performance. When in the condition of low demanded performance, ease secondary indicators appropriately to get low cost and high efficiency. Finally, strive for the less types of materials and additives, the more convenient operation, and the lower cost.

\section{(3) Design flow.}

Choose the fluid materials and additives $\rightarrow$ decide the specifications and performance parameters $\rightarrow$ design of multirecipe $\rightarrow$ make the experimental comparison $\rightarrow$ decide the preparation, maintenance and adjustment schemes $\rightarrow$ draw up 
circulation, purify, management measures $\rightarrow$ decide the fluid materials and additives $\rightarrow$ drilling applications $\rightarrow$ modify the recipes and experiments.

There is an uncertain condition under the soil. Before drilling, we can't forecast the situations. For example, the change of rocks, fault zones, formation pores, and karst caves. All of above need to adjust the drilling fluid in time, which can ensure the drilling security and stability. The qualified drilling fluid equals to the safe, stable and highefficient drilling.

(4) The quality control for experiments of fluid recipe

Take tests according to the standard practice for testing.

Stratum samples must be unstable and representative.

Equipments and measuring instruments must be intact and accurate.

Improve the accuracy of test by repeating and contrasting experiments and adjust it to the practical accuracy on machines.

According to the fluid types and performance pedigree of different hole sections, take contrast experiments and orthogonal experiments to calculate the cost and write out the fluid recipe which meets the need of design and cost.

\subsection{The fluid quality control in the drilling stage}

\subsubsection{The quality control in the management of materials and additives}

(1) Sort all the materials and additives in the field and put them in tidy, prohibiting material mixes. Materials out of expiry date mustn't be used. Put something on the top and under the materials. Prevent them from the damp and rain.

(2) All the materials in the field must come from the regular factories and have the approved certificates. After the strict test, all the materials can be carried into the field. Take experiments and analysis on the grouting material regularly.

(3) Build a management system with the material plans, tests, daily management and statistics, and take it as a branch system of the whole drilling management system.

3.3.2 The quality control of setting the fluid circulation system

The fluid circulation system controls the drilling fluid solid content. The common solid control contains the mechanical removal, chemical flocculation and settling sand removal. There are great differences among the drilling purpose, the formation characters and the drilling methods, but the fluid circulation system can't meet all the needs. According to the low cost and the easy operation, only meet the hole security and the fluid function to dispose the fluid circulation system. Such as the mechanical removal, chemical flocculation and settling sand removal. If the first one can solve the problem, the rest are not used.

Setting the fluid circulation system has an important effect on the fluid service cycle. The efficient circulation system can control the fluid solid content and Maintain the fluid performance parameters, which can increase the fluid utilization and decrease the drilling cost. The quality control of the fluid circulation system is set from the following two points: the circulatory system ancillary equipment and setting of circular pool and groove. The specific structure chart is shown in Picture 3.

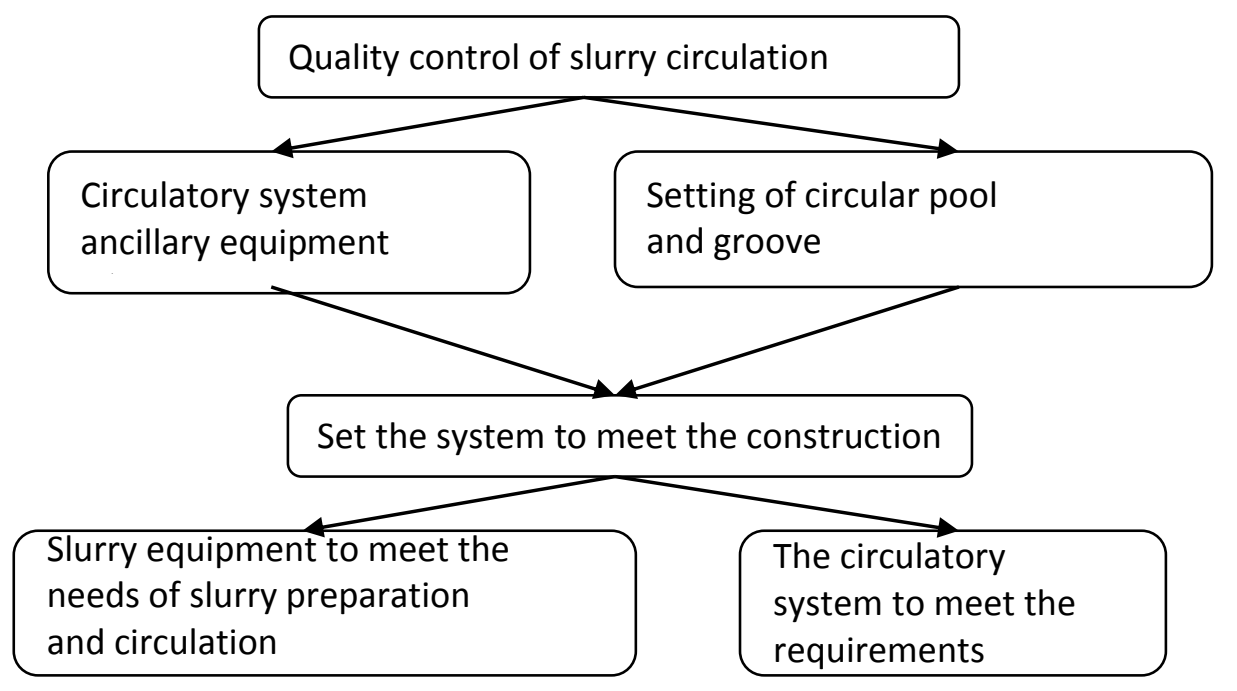

Picture 3. Structure chart of the quality control of the slurry circulation system

\subsubsection{The quality control of the fluid allocation and maintenance}

(1) Allocate the fluid according to the design. The fluid recipe and methods can't be changed unless the related technical personnel agree to change.

(2) Test the fluid performance indicators according to the design requirements at regular time and add the fluid primer in time to maintain the stability. 
(3) Control the addition of the fluid primer according to the design and decide the orders and methods according to the types of the fluid primer.

(4) After adding the primer while mixing, control the mixing time to ensure all kinds of primer mixed uniformly. The time depends on the slurry recipe. As for the emergency, for example, the leakage, decrease the mixing time in real conditions.

(5) According to different formation characters, adjust the performance timely to meet the drilling needs.

(6) Clean the circular groove and the sedimentation basin at regular time to prevent rain and water from inflowing into the circulation system.

(7) The waste water and slurry can't inflow into the slurry pool while injecting holes with the cement, especially the fluid with the organic agent.

\section{Conclusion}

This passage talks about the composition of the drilling fluid quality control system and how to ensure the drilling fluid quality, and gives a series of principles, methods and requires of the drilling fluid quality control for discussing. For the drilling of the mine geological coring, because of the full use of lines for core-drilling technology, solid-free fluid and solid-low slurry are mainly used in machines. Prepared application schemes mainly come from the product business. There are not enough types of production and more accurate measurement and test equipment, which can't prepare and adjust the fluid in a scientific way and is lack of markets for asking the drilling technology. Many machines can't meet the need of the drilling of complicated formations. We all hope that there is a kind of intelligent "expert system for drilling slurry of geological core" using in machines, which greatly improve the drilling level of professional technique.

\section{References:}

[1] Li Xudong. Study on the Importance of Meticulous Management for Construction Project [J]. Oriental Enterprise Culture. 2014(03)

[2] Hu Jiliang, Tao Shixian. The Design Factors and Analysis of Deep Geological Drilling Fluid Systems [J]. Prospecting Engineering (Geotechnical Drilling and Excavation Engineering). 2011(4) of Volume 38

[3] Yuan Mingwen, Yao Gaiwei. Reflections and Recommendations on Improving the Quality of the Deep Drilling Core [J]. Pioneering With Science \& Technology Monthly. 2015(07)

[4] Qin Chengchun. Discussion on Improving the Quality of Deep Drilling Coring Techniques [J]. West-China Exploration Engineering.2016(01)

[5] Peng Xudong, Chen Liyi, He yuanxin, Zhu Wenjian. Realization of Expert System of Drilling Slurry at the Scene [J]. Prospecting Engineering (Geotechnical Drilling and Excavation Engineering).2013 Supplement of Volume 40

[6] Zhu Hengyin, Cai Zhengshui, Wang Qiang, Cheng Hongwen, Zhang Zheng. Study on Deep Drilling Techniques and Applications [J]. Equipment for Geotechnical Engineering.2013(06)

[7] Liu Shunquan, Song Dongyong, Zhang Xiaoli, Bao Zhiqiang. Application of Treatment Technology of Waste Drilling Fluid in 96 - 13 Storage Wells While Drilling [J]. Natural Gas Technology and Economy.2014(01)

[8] He Long, Lin Xuanyi, Fang Yongchun, Cheng Shaojun, Li Jianhua. Thought and Practice of Treatment Technology of Waste Drilling Fluid in Oil Field [J]. Petrol \& Chemical Equipment.2013(06)

[9] Chen Bo, Zheng Jie. Innoxious Treatment Technology and Application of Wasted Drilling Fluid in Northwest Sichuan Region [J]. Inner Mongolia Petrochemical Industry.2010(17)

[10] Chen Xiaoying. Innoxious Treatment of Wasted Drilling Fluid and the Environment [J]. Science and Technology Innovation Herald.2009(21)

[11] Liu Dawei, Bai Juan. Disposal Countermeasures of Drilling Fluid in Loess Zone. 2011

[12] Deng Hongzhang, Wang Yanhua. Study on CMC Method of Synergetic Degradation in Waste Drilling Fluid.2011

[13] Liu Dawei, Bai Juan. Disposal Countermeasures of Drilling Fluid in Loess Zone.2011

[14] Deng Hongzhang, Wang Yanhua. Study on CMC Method of Synergetic Degradation in Waste Drilling Fluid.2011 